\title{
Effects of alcohol on the partial reinforcement effect
}

ANTHONY TAYLOR, ROBERT LEHR, DAVID F. BERGER AND CYNTHIA A. TERRY, STATE UNIVERSITY OF NEW YORK, College at Cortland

The hypothesis was tested that partial reinforcement results in increased resistance to extinction because stimuli produced by fractional anticipatory frustration responses $\left(\mathrm{r}_{\mathrm{F}}\right)$ become conditioned to adient instrumental responses during training. On the assumption that alcohol diminishes or inhibits $r_{F}$ the drug was administered during training trials. Extinction findings failed to support the hypothesis.

Amsel (1958) accounts for the Partial Reinforcement Effect (PRE) by suggesting that partially reinforced (PR) Ss during training are subject to a frustration reaction (F) in the goal area on nonreward trials, and that as training under a PR schedule continues fractional representations of $F\left(r_{F}\right)$ are elicited by stimuli regularly preceding arrival at the goal. In addition, the stimulus consequences of $r_{\mathrm{F}}\left(\mathrm{s}_{\mathrm{F}}\right)$ gradually become conditioned to the goal-approaching responses $\left(R_{\mathbf{a}}\right)$; and it is this latter bond $\left(s_{\mathrm{F}} \rightarrow R_{a}\right)$ that accounts for the superior resistance of $P R$ Ss to subsequent extinction.

The most obvious approach to testing Amsel's foregoing analysis would be to manipulate $r_{1}$ and observe any corresponding changes in the magnitude of the PRE. Previous research by Barry, Wagner \& Miller (1962) suggests that alcohol acts selectively on $r_{\mathrm{F}}$ to suppress or diminish it. Accordingly, the strategy of the present study was to assume that alcohol does have an inhibiting effect on $r_{1}$, and to determine whether the administration of the drug to PR rats prior to daily training in a straight runway has the effect of facilitating subsequent extinction.

The foregoing study by Barry et al (1962) is of interest also in supporting the general proposition implied by the suggested relationship between $r_{1}$ and the PRE: that $r_{F}$ is responsible at least in some measure for the phenomenon of experimental extinction. Operating on the assumption that alcohol and sodium amytal inhibit $r_{F}$, Barry et al found that the administration of either drug during extinction had the effect of retarding the extinction process. It should be noted explicitly that the current study in a real sense constitutes an extension of the research by Barry and his associates.

While Amsel's theory predicts that the inhibition of $r_{1}$ during the extinction stage would retard extinction, the theory also predicts that the inhibition of $r_{F}$ in $P R$ Ss during the acquisition stage would facilitate extinction. Therefore, in the present study confounding between the possible effects of alcohol during acquisition and during extinction was avoided by discontinuing the alcohol and placebo injections with the onset of the extinction stage.

Method. The Ss were 44 male hooded rats from the Syracuse University breeding colony, ${ }^{1}$ 184-200 days old on the first day of training. Ten Ss were discarded, nine because of death or illness and one because of training difficulties.

The apparatus was a $70 \mathrm{in}$. straight runway $3 \mathrm{in}$. wide, $4 \mathrm{in}$. high, painted flat black and covered with clear, hinged Plexiglas. The first 18 in. and last 18 in. of the runway served as start box and goal box, respectively, and they were separated from the 34 in. alley by black Masonite guillotine doors. In the goal box a white metal food cup was centered against the rear wall.

The two latency measures recorded on $.01 \mathrm{sec}$ clocks were: starting time from the opening of the start box door to a photoelectric beam 5 in. into the alley, and running time from the first beam to a second beam 24 in. from the former beam.

The $S s$ were run in scjuads of 20 and 24,23 days apart. The Ss in each squad were randomly assigned in equal numbers to four treatment groups. A $23 \mathrm{~h}$ food deprivation schedule with $1 \mathrm{~h}$ ad lib feeding was introduced 14 days prior to the first training day and maintained throughout the experiment. The Ss were adapted to the apparatus and trained to eat reward pellets on the last two and three days, respectively. There were 52 training trials: one trial on the first and second days, two trials on the third day, and six trials on each of the subsequent eight days. Then six extinction trials per day followed for the next five days. The four groups received different combinations of the two independent variables: solution (alcohol vs isotonic saline) and reinforcement schedule (100\% vs $50 \%$ ). The PR schedule was random with the following restrictions: the first four trials were reinforced, while three trials were reinforced with each successive six-trial block. These restrictions assured a fairly even distribution of reinforced trials over the span of training.

Each squad was divided into subsquads of four Ss, each S having been randomly assigned to a different treatment group. One subsquad at a time was run, with each $S$ running one trial on each turn. The intertrial interval ranged from approximately $1 \mathrm{~min}$ to 6 min, depending on the other $S s$ in the subsquad and the point in training or extinction. Approximately $10 \mathrm{~min}$ before a subsquad began daily training, intraperitoneal injections of either alcohol (15 ml/kg of a $10 \%$ solution in isotonic saline) or isotonic saline $(15 \mathrm{ml} / \mathrm{kg}$ ) were administered. On a given trial Ss were taken directly from the home cage and placed in the start box, and after 5 sec the start box door was opened. On reward trials Ss were removed from the goal box immediately after consuming the five reward pellets $(.045 \mathrm{~g}$ each). On nonreward trials both during acquisition and extinction $S s$ were confined to the goal box for 15 sec. A trial was discontinued if Ss failed in 2 min either to progress to the initial photoelectric beam, to reach the second beam after interrupting the first beam, or to enter the goal box after breaking the second beam. Also, Ss were removed from the goal box after $60 \mathrm{sec}$ of failing to eat all the reward pellets. Immediately after all Ss had completed daily training they were injected with saline if they had received alcohol prior to running, and they received alcohol if they had received saline before running. These second daily injections had the effect of holding constant across all treatment groups any cumulative effects due to either or both solutions.

Results and Discussion. To facilitate statistical analyses by having an equal number of Ss in each group $(n=9)$, data for one additional $\mathrm{S}$ was provided by mean values being added to the Alcohol-50\% and Placebo-100\% groups. For each S mean reciprocal values (1/latency) were calculated for starting time and running time based on the first four acquisition trials, and each subsequent block of six acquisition and extinction trials. Then appropriate factorial analyses of variance were computed using these mean values.

The findings for starting time and running time during the extinction stage were very similar, and for that reason only the trends in running time are presented graphically (Fig. 1). Analyses

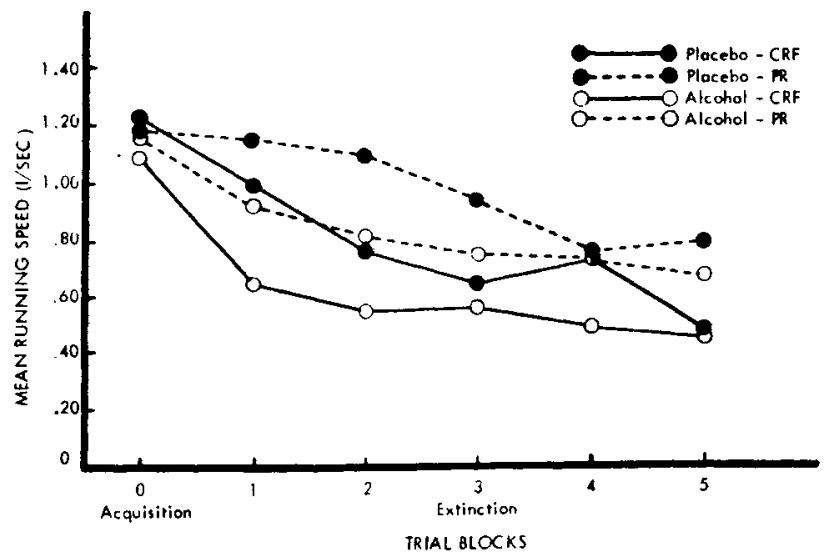

Fig. 1. Mean running speeds in blocks of six trials for the four experimental groups during the last six training trials and during ext inction. 
of variance of data from the last block of six acquisition trials through the entirety of extinction revealed significant schedule effects for both starting time $(F=6.73, \mathrm{df}=1 / 32, \mathrm{p}<.05)$ and running time $(F=9.61, d f=1 / 32, p<.01)$, with the $P R S s$ running faster than the continuous reinforcement (CRF) Ss. There was no interaction between schedules and solutions as would be expected if, in fact, $r_{F}$ is responsible for the greater resistance of PR Ss to extinction $(F<1.00)$. Thus, the findings of the present study do not support Amsel's proposal nor are they congruent with the previously cited findings by Barry and his associates.

Of course, the present evidence against Amsel's explanation for the PRE rests upon the validity of the assumption that alcohol inhibits $r_{F}$. Two considerations tend to contraindicate the additional possibility that alcohol does suppress $r_{F}$ but only in dosages greater than those used in the current study. First, it would be suspected that if the dosage level was potent enough to result in certain "stimulus changes," then it would act on $r_{F}$ also. Evidence for stimulus change may be deduced by comparing acquisition and extinction data. In acquisition there was no main effect of solutions in starting time $(F=1.10$, df $=1 / 32, p>.05)$ or running time $(F<1.00)$. In contrast, there was a significant solutions effect in extinction running time $(F=6.59, \mathrm{df}=1 / 32$, $\mathrm{p}<.025$ ) and a similar though nonsignificant solutions effect in extinction starting time $(F=3.41, \mathrm{df}=1 / 32, p<.10)$. The fact that each group previously receiving alcohol ran appreciably slower than the group having had saline and the same reward schedule would seem to be indicative of stimulus generalization decrement occurring as a result of a cessation of alcohol intoxication. The second argument against the notion of impotent dosages being used is that for the alcohol injections the concentration and volume-body weight formula were identical to those ostensibly effective in dissipating the anxiety associated with conflict (Barry \& Miller, 1962).

A brief further consideration of the available acquisition data is warranted in view of the opportunity to examine the efficacy of the $r_{F}-s_{F}$ construct in yet another respect. By way of background, Amsel (1958) asserts that $s_{F}$ : not only becomes linked with $R_{a}$, but also has motivational properties that have the effect of increasing general drive level. The finding that PR Ss achieve greater asymptotic running speeds than CRF Ss (e.g., Goodrich, 1959) is explained by Amsel in terms of the motivational advantage of PR Ss experiencing $\mathrm{s}_{\mathrm{F}}$. By taking into account that CRF Ss are energized to a greater extent than PR Ss by stimuli produced by anticipatory goal responses, Wagner (1963) subsequently deduced that if sodium amytal selectively inhibits $r_{F}$ (and hence $s_{F}$ ), then the usual asymptotic difference in latency should be reversed. While Wagner found support for his prediction administering 64 training trials, the present study found no significant effects due to the different reward schedules over 52 trials $(F<1.00)$. Furthermore, while graphic inspection suggested asymptotic performance was not reached, inspection also failed to disclose any meaningful group differences beginning to emerge.

\section{REFERENCES}

AMSEL, A. The role of frustrative nonreward in noncontinuous reward situations. Psychol Bull., 1958, 55, 102-119.

BARRY, H.,III, \& MILLER, N. E. Effects of drugs on approach-avoidance conflict tested repeatedly by means of a "telescope alley." $J$. comp. physiol Psychol, 1962, 55, 201-210.

BARRY, H., III, WAGNER, A. R., \& MILLER, N. E. Effects of alcohol and amobarbital on performance inhibited by experimental extinction. $J$. comp. physiol Psychol, 1962, 55, 464-468.

GOODRICH, K. P. Performance in different segments of an instrumental response chain as a function of reinforcement schedule. J. exp. Psychol, $1959,57,57-63$.

WAGNER, A. R. Sodium amytal and partially reinforced runway performance. J. exp. Psychol., 1963, 65, 474-477.

\section{NOTE}

1. The authors are indebted to Drs. Wallace R. and Dorothy E. McAllister for generously providing the rats for the study. 